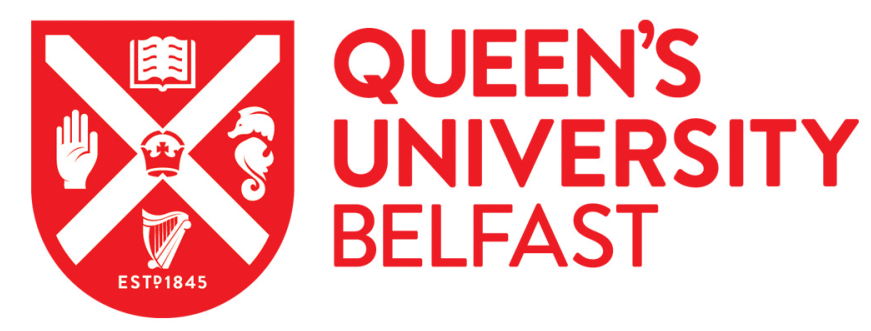

\title{
Understanding the Interaction between Low-Energy Electrons and DNA Nucleotides in Aqueous Solution
}

McAllister, M., Smyth, M., Gu, B., Tribello, G. A., \& Kohanoff, J. (2015). Understanding the Interaction between Low-Energy Electrons and DNA Nucleotides in Aqueous Solution. Journal of Physical Chemistry Letters, 6(15), 3091-3097. https://doi.org/10.1021/acs.jpclett.5b01011

Published in:

Journal of Physical Chemistry Letters

Document Version:

Peer reviewed version

Queen's University Belfast - Research Portal:

Link to publication record in Queen's University Belfast Research Portal

\section{Publisher rights}

This document is the Accepted Manuscript version of a Published Work that appeared in final form in

The Journal of Physical Chemistry Letters, copyright @ 2015 American Chemical Society after peer review and technical editing by the publisher.

To access the final edited and published work see http://pubs.acs.org/doi/abs/10.1021/acs.jpclett.5b01011

\section{General rights}

Copyright for the publications made accessible via the Queen's University Belfast Research Portal is retained by the author(s) and / or other copyright owners and it is a condition of accessing these publications that users recognise and abide by the legal requirements associated with these rights.

Take down policy

The Research Portal is Queen's institutional repository that provides access to Queen's research output. Every effort has been made to ensure that content in the Research Portal does not infringe any person's rights, or applicable UK laws. If you discover content in the Research Portal that you believe breaches copyright or violates any law, please contact openaccess@qub.ac.uk. 


\title{
Understanding the Interaction between
}

\section{Low-Energy Electrons and DNA Nucleotides in Aqueous Solution}

\author{
Maeve Mc Allister, ${ }^{\dagger}$ Maeve Smyth, ${ }^{\dagger}$ Bin Gu, ${ }^{\dagger, \ddagger}$ Gareth A. Tribello, ${ }^{*, \dagger}$ and Jorge \\ Kohanoff ${ }^{\dagger}$ \\ Atomistic Simulation Centre, School of Mathematics and Physics, Queen's University \\ Belfast, Belfast, BT7 1NN, and Department of Physics, Nanjing University of Information \\ Science and Technology, Nanjing 21004, China \\ E-mail: g.tribello@qub.ac.uk
}

\begin{abstract}
Reactions that can damage DNA have been simulated using a combination of molecular dynamics and density functional theory. In particular, the damage caused by the attachment of a low energy electron to the nucleobase. Simulations of anionic single nucleotides of DNA in an aqueous environment that was modelled explicitly have been performed. This has allowed us to examine the role played by the water molecules that surround the DNA in radiation damage mechanisms. Our simulations show that hydrogen bonding and protonation of the nucleotide by the water can have a significant effect on the barriers to strand breaking reactions. Furthermore, these effects are not the same for all four of the bases.
\end{abstract}

Keywords: DNA damage, computer simulation, free energy, DFT

\footnotetext{
*To whom correspondence should be addressed

†Queen's University Belfast

${ }^{\ddagger}$ Department of Physics, Nanjing University of Information Science and Technology, Nanjing 21004, China
} 


\section{Introduction}

Ionising radiation can damage the DNA of living cells in a variety of ways. This damage can cause mutations that lead to diseases such as cancer years after exposure. ${ }^{1}$ The effect radiation has on biological tissue has been harnessed in the treatment of cancer, where radiation therapy targets tumorous cells with the aim of killing them. There has been sustained interest, during the past decade, in the molecular level details of the damage process, with a view to understanding and further enhancing the efficacy of treatments.

Ionising radiation can damage DNA in a cell directly, ${ }^{2,3}$ or it can excite molecules in the cellular surroundings. These excited state species can go on to interact with DNA strands and cause damage. ${ }^{4}$ It is common to examine the DNA damage process in water as it is the principle component of the cell ${ }^{5}$ and because the radiochemistry of water is reasonably well known. ${ }^{6,7}$ Whether it is the low-energy electrons (LEE) or the $\mathrm{OH}$ radicals that are generated by the radiolysis of water that cause the damage to DNA is less clear and is difficult to investigate experimentally. Sanche et al, ${ }^{8,9}$ have demonstrated that electrons with energies as low as $0 \mathrm{eV}$ can cause strand breaks in dry DNA. Nguyen et al ${ }^{10}$ have shown that the low energy electrons produced following the radiolysis of water, cause the most damage to aqueous DNA. In particular, their results suggest that reductive damage caused by low energy electrons lead to more strand breaks than oxidative damage caused by $\mathrm{OH}^{*}$ radicals. However, experiments have also highlighted the pronounced effect the environment can have on the LEE damage process in DNA ${ }^{11-13}$ but results on the role played by water are mixed. Structural waters appear to reduce the damage, ${ }^{13}$ while adding bulk water increases the damage done by LEEs. ${ }^{12}$ These water molecules can affect the damage process by (a) modifying the effective potential felt by the excess electron and (b) influencing the dynamics of any dissociated fragments.

The mechanism via which low-energy electrons cause DNA strand breaks has been stud-

ied extensively. ${ }^{14-20}$ The electron attachment process is generally assumed to happen via electronic resonances at positive energies to form metastable states called transient negative 
ions (TNI). These species can survive long enough to allow for energy transfer to vibrational motion to occur. This transfer process can lead to dissociative electron attachment (DEA). Whether a molecule will dissociate or not is dependent on the energetic barrier for dissociation and on the energy difference between the resonant state and the ground state of the anion.

Clearly, conventional ground state electronic structure methods, which are suitable for the study of large molecules and for the explicit treatment of the environment, cannot be used to study processes such as TNI formation and DEA. At the same time, however, Rmatrix calculations, ${ }^{21}$ although well-suited to the study of DEA in molecular targets, are very challenging for large molecules such as full nucleotides. ${ }^{22}$ In addition, such calculations do not provide information on the dynamics of the dissociated fragments. Preliminary simulations of DEA-like dissociation in solvated nucleobases indicate that there is a pronounced caging effect, which causes the anion to transfer its excess energy to the environment and then reform. ${ }^{23}$ We have thus assumed in this work, as is common in a number of other papers, that after a relatively short equilibration, the excess electron settles in the LUMO of the system, which is generally located in the DNA nucleobase. This means that the problem is thus now one of determining the ground state electronic structure for a solvated anion and the thermally activated processes that this species can undergo.

A series of recent studies have demonstrated that water molecules in the solvation environment ${ }^{17,24,25}$ can have a significant effect at the level of resonances, ${ }^{26}$ caging $^{23}$ and groundstate thermodynamics. ${ }^{27-30}$

These studies have shown that the ease of the strand breaking reaction is dependent on the particular nucleotide and that the barrier to strand breaking is significantly higher when the DNA is solvated. In addition, there has been a suggestion that the DNA anion can react with the surrounding water molecules and become protonated and that furthermore this has an effect on the size of the barrier. This is in agreement with some experimental observations that would seem to suggest that protonation of DNA anions can prevent strand breaks. ${ }^{31}$ 
In this work we thus chose to systematically investigate the ease with which the various ground-state anions of the DNA bases will protonate and the effect this protonation has on the thermally-activated strand breaking reaction. We found that most of the anions are highly likely to protonate and that this can have a significant and perhaps under appreciated effect on the reactivity of DNA.

\section{Methodology}

We simulated each of the DNA nucleotides, Adenosine (dAMPH), Cytidine (dCMPH), Thymidine (dTMPH) and Guanosine (dGMPH) monophosphates, separately using a combination of molecular dynamics (MD) and density-functional theory (DFT). Each of these molecules were placed in a cubic box of $15 \AA$ that was filled with 100 explicit DFT water molecules. All simulations were run using CP2K and the ab initio Quickstep (QS) module. ${ }^{32}$ Energies and forces were calculated using the PBE functional ${ }^{33}$ in conjunction with GTH pseudo potentials, ${ }^{34}$ and a TZVP-GTH basis set. An additional electron was introduced and unconstrained MD was performed for 6.5 ps in order to allow the anion to equilibrate to a low-energy configuration. During these MD simulations temperature was fixed at $300 \mathrm{~K}$ using a CSVR thermostat with a relaxation time of 0.1 ps. The strand breaking and protonation reactions are activated processes that will not be observed on the simulation timescale

so they were investigated using the Blue Moon ensemble approach. ${ }^{35}$ In all these calculations the constraints were placed on an inter-atomic distance and were used to accelerate the formation and cleavage of chemical bonds.

\section{Results and discussion}

The aqueous environment around a DNA strand will screen the electrostatic interactions between charges. This effect can be easily incorporated into any model by using a polarizable continuum. However, water can also form hydrogen bonds to the DNA potentially stabilising 
high energy anions. It can also react with the DNA and cause it to become protonated. To study these two effects we thus chose to investigate single DNA nucleotides dissolved in water molecules that were modelled explicitly in a similar manner to Smyth and Kohanoff. ${ }^{29}$ They initially simulated the neutral system and then vertically attached an electron to the base. We took the configurations from their work and then relaxed the solvated anion for a further 6.5 ps. This relaxation gave the hydrogen bonding network sufficient time to adapt to this more highly charged solute molecule and, by the end of the relaxations, we found that the water molecules around the base had rearranged themselves so as to form explicit hydrogen bonds between the solvent and the solute. The shortest hydrogen bonds that formed during these relaxations are shown in figure 1. Furthermore, the $\mathrm{dAMPH}^{-}$was found to spontaneously protonate at the site shown in figure 1 during relaxation. To test whether adding an additional electron to the base promotes hydrogen bond formation we took the final configuration from our simulations, removed the electron and re-equilibrated. We found that in all the bases the hydrogen bonds shown in figure 1 broke during these simulations, which does indeed suggest that these bonds only form when the additional electron is present.

To investigate how the charged nucleotide decomposes we ran a number of simulations using the Blue Moon ensemble. In these MD simulations a Lagrange multiplier was used to constrain an interatomic distance to a particular value. The average value of the Lagrange multiplier in such simulations is equal to the potential of mean force associated with the constraint. Hence, if we calculate this quantity for a number of intermediate distances between having the bond formed and broken, we can determine the free energy change associated with breaking the bond using numerical integration.

The first reaction that we studied using this technique was the protonation of the base at the sites shown in figure 1. In these calculations the distance between the electronegative atoms highlighted in figure 1 and the hydrogen atom on the nearest water molecule was used as a CV. The free energies obtained are shown in figure 2 together with error bars that were calculated using block averaging. Protonation is clearly favourable for $\mathrm{dCMPH}^{-}$ 
and $\mathrm{dTMPH}^{-}$and unfavourable for $\mathrm{dGMPH}^{-}$. The protonation of $\mathrm{dAMPH}^{-}$is barrier-less, which goes some way towards explaining why this base protonates spontaneously in unbiased MD simulations. $\mathrm{dCMPH}^{-}$and $\mathrm{dTMPH}^{-}$, meanwhile, have two minima that are separated by a barrier that is less than $1 \mathrm{kcal} \mathrm{mol}^{-1}$. In one of these minima the proton is bonded directly to the electronegative atom, while in the other the proton is covalently attached to the water and hydrogen bonded to the electronegative atom. Protonation of $\mathrm{dGMPH}^{-}$is not favourable as for this base there is no minimum in the free energy surface for structures that have the excess proton covalently bound to the base. This result is in agreement with those discussed in ${ }^{36}$ where it is suggested that the guanine radical anion is deprotonated at ambient temperature in the crystal structure.

Our simulations have shown that upon electron attachment, protonation is likely for many of the bases. This effect has not been included in many of the calculations that have thus far been performed on the strand breaking (damage) reactions although Smyth and Kohanoff ${ }^{29}$ have suggested that the barrier to the strand breaking reaction increases when the base is protonated. To examine whether this was the case we ran two sets of Blue Moon simulations for each of the nucleotides. In these calculations two restraints were used, the first of which was on the length of the $\mathrm{C}_{3}$,- $\mathrm{O}_{3}$, bond. Multiple simulations were performed with this bond length constrained to a number of different values in order to extract the free energy profiles shown in figure 3. Two calculations at each of these bond lengths were performed. In the first of these the distance between the electronegative atom highlighted in figure 1 and the hydrogen atom on the nearest water molecule was constrained to be $1.8 \AA$ which we found from unconstrained simulations was the typical distance between the electronegative site and the non-covalently-bound hydrogen in a basewater hydrogen bond. In the second of these calculations this same distance was constrained to be $1.05 \AA$ which our unconstrained simulations told was the typical length of the covalent bond between the base and the protonating hydrogen atom. The results from these two sets of calculations were analysed separately in order to extract the free energy profiles for the 
strand breaking reaction in the protonated and deprotonated configurations. Figure 3 shows that the protonation of the base has a significant effect on free energy of reaction. This reaction is free-energetically-unfavourable whenever an additional proton is attached to the base. Furthermore, the barrier to reaction increases for all the bases apart from dAMPH ${ }^{--}$ upon protonation. For $\mathrm{dCMPH}^{\cdot-}$ this effect is particularly significant. $\mathrm{dAMPH}^{--}$behaves differently to the other bases - the barrier to the strand breaking reaction is $4 \mathrm{kcal} \mathrm{mol}^{-1}$ lower when the system is protonated but is similar to the barriers for the deprotonated versions of the other bases. $\mathrm{dAMPH}^{-}$is the base that protonates spontaneously in unbiased MD simulations and for which, figure 2 would suggest the deprotonated configuration in which the electronegative atom is a hydrogen bond acceptor, is not stable. Intuitively, one would expect the barrier to increase for the protonated system as the addition of the proton would stabilise the negatively charged anion. The fact that singly protonating $\mathrm{dAMPH}^{-}$lowers the barrier to the reaction may simply be a consequence of the fact that there is no truly stable configuration of this system in which there is a hydrogen bond between the electronegative atom and a water molecule. We thus examined what effect a second protonation would have on the barrier to the strand breaking reaction. The site at which the second proton was added is shown circled with blue dashed line in figure 1 . The top right panel of figure 3 shows that the barrier to the strand breaking reaction increases in this doubly protonated system in agreement with our hypothesis.

The results shown in figure 3 connect what is observed in experiments with findings from simulations. Our results suggest that understanding whether or not the negatively charged nucleotide will protonate is highly important as protonation can have a significant effect on both the ease of the strand breaking reaction and the position of the equilibrium. To give an example of how significant protonation effects can be, consider the recent article by Wang et al. ${ }^{37}$ They showed that strand breaking on a DNA strand is significantly less likely to occur at Cytosine sites. If one only examines the free energy barriers for the deprotonated systems there is no explanation for this result - the barriers to the strand breaking reaction 
in the deprotonated bases are all reasonably similar. However, the barrier to the strand breaking reaction is significantly higher for protonated Cytosine, which together with the result in figure 2 that tells us that it is easy to protonate this base, helps explain Wang's results. Furthermore, the fact that the protonated $\mathrm{dCMPH}^{--}$ion is stable in our results is in agreement with theoretical results from $\mathrm{Gu}$ et al. ${ }^{31}$ who obtained similar results in geometry optimisation and energy minimisation calculations using the B3LYP functional in Gaussian03. It also agrees with $a b$ initio simulation results performed using a continuum solvation model by Naumov and von Sonntag ${ }^{38}$ who argued based on energetic considerations that the Cytosine radical anion should protonate quickly in water. In fact, it is known from experiment ${ }^{39}$ that the Cytosine radical anion is so basic (its pKa is 13 as opposed to 10 in all other bases) that when this species appears in a DNA double helix it will extract the proton from its complementary Guanine to form a protonated Cytosine and deprotonated Guanine. ${ }^{40}$

There is a considerable amount of evidence that suggests that strand breaks are most likely to occur at the Guanine site on the DNA strand. ${ }^{17,27,37,41-44}$ In particular, Wang et $a l^{37,41}$ have found that dissociation by the addition of electrons to the Guanine base, results in significant quantities of single and double strand breaks. The fact that strand breaks are likely to occur at Guanine sites is in agreement with the results shown in figure 3. Figure 2 shows that Guanine will be deprotonated, while figure 3 shows that the barrier for breaking the $\mathrm{C}_{3},-\mathrm{O}_{3}$, bond in the deprotonated $\mathrm{dGMPH}^{-}$is only $19 \mathrm{kcal} \mathrm{mol}^{-1}$ and that the total free energy change upon reaction is approximately zero.

Our results show that strand breaking reactions are more likely to occur at the purines $\left(\mathrm{dGMPH}^{-}\right.$and $\left.\mathrm{dAMPH}^{-}\right)$than they are at the pyrimidines. Figure 2 shows that Adenine will most likely be protonated, while figure 3 shows that the barrier to the strand breaking reaction in protonated $\mathrm{dAMPH}^{-}$is $18 \mathrm{kcal} \mathrm{mol}^{-1}$. In addition, the free energy change for the Adenine strand breaking reaction is considerably less positive than the free energy change for both the Cytosine and Thymine strand breaking reactions. This difference we observe in the 
propensity for each of the bases to undergo strand breaking is consistent with experimental work by Zheng et $a l^{43}$ who observed that the extent of damage to short polynucleotide sequences (GCAT) is lower when Guanine and Adenine are absent.

Previous theoretical works ${ }^{15,17,45}$ have focussed on the breaking of the $\mathrm{C}_{3}$,- $\mathrm{O}_{3}$, bond as experiments have seemed to suggest that this is the most likely bond to break in the DNA strand. ${ }^{43,46,47}$ We chose to also investigate the glycosidic bond to see if the results from our models are in agreement with these observations. Similarly to the calculations that were performed on the breaking of the $\mathrm{C}_{3}$,- $\mathrm{O}_{3}$, bond we ran Blue Moon ensemble calculations to determine the barrier to the glycosidic bond breaking reaction in both the protonated and deprotonated nucleotides. The results from these calculations are shown in figure 4. Once again we find that the free energy changes for the strand breaking reactions are all positive and hence that the strand breaking reaction is unfavourable. For both purines, the barrier for breaking the glycosidic bond is about $5 \mathrm{kcal} \mathrm{mol}^{-1}$ higher than the barrier for breaking the $\mathrm{C}_{3}$,- $\mathrm{O}_{3}$, bond. In doing this comparison we compare the barriers for protonated $\mathrm{dAMPH}^{--}$and deprotonated $\mathrm{dGMPH}^{-}$as these nucleotides will most likely be in these forms in solution. Intriguingly, the barriers for breaking the glycosidic bonds in the pyrimidines are similar $\left(\mathrm{dTMPH}^{-}\right)$and slightly lower $\left(\mathrm{dCMPH}^{-}\right)$than the barriers for breaking the corresponding $\mathrm{C}_{3}$,- $\mathrm{O}_{3}$, bonds. When damage occurs at these bases one should therefore expect similar numbers of broken glycosidic and $\mathrm{C}_{3}{ }^{-}-\mathrm{O}_{3}$, bonds. This is precisely what has been observed by $\mathrm{Gu}$ et $a l^{31}$ who demonstrated that, after electron attachment to protonated Cytosine, $\mathrm{N}-\mathrm{C}$ bond cleavages are more abundant than C-O bond cleavages. Nevertheless, the fact remains that the most important damage reactions to DNA involve $\mathrm{C}_{3},-\mathrm{O}_{3}$, cleavage. Our results suggest that this is because the easiest bonds to break are the $\mathrm{C}_{3},-\mathrm{O}_{3}$, bonds involving the purines. The barriers to all strand breaking reactions are higher for the pyrimidines and these reactions are thus less likely. 


\section{Conclusion}

The simulations we have performed have demonstrated that the aqueous environment plays a significant role in DNA damage reactions. Hydrogen bonds can form between the base and the surrounding water molecules and these serve to stabilise the anion. These results are in agreement with previous results from Kumar and Sevilla ${ }^{48,49}$ who showed that the barrier to dissociation of a $\mathrm{dTMPH}^{-}$ground-state, radical anion was increased when hydrogen bonding effects from a microsolvated environments were included. We have further shown that, as well as hydrogen bonding to the base, water molecules can donate protons to the base. This thermally-activated reaction is favourable for all bases other than Guanine. The manner in which protonation and hydrogen bonding affects the behavior of anions in excited states is a matter of current investigation. What is clear from this work, however, is that protonating the anionic nucleobase when it is in the ground state makes the thermally-activated strand breaking reaction unfavourable. The solvent thus has a significant effect and one that cannot be easily incorporated into continuum solvation models.

Surprisingly we have found that there are marked differences between the various different bases. The purines have the lowest barriers to strand breaking reactions and will break at the $\mathrm{C}_{3},-\mathrm{O}_{3}$, bond. Pyrimidines, meanwhile, have higher barriers to reaction but the barriers for breaking the $\mathrm{C}_{3}$,- $\mathrm{O}_{3}$, bond and the glycosidic bond are similar. How these differences will manifest themselves in longer single stranded DNA or in double stranded DNA, where the bases are less solvent accessible, is interesting and will be the subject of future studies.

\section{Acknowledgement}

The authors would like to thank Leon Sanche, Anil Kumar and Amit Adhikary for reading an early draft of the manuscript and providing useful comments. The simulations were run in the UK national facility ARCHER, through the UKCP consortium funded by EPSRC grant EP/K0139459/1. 


\section{References}

(1) Baccarelli, I.; Bald, I.; Gianturco, F. A.; Illenberger, E.; Kopyra, J. Electron-induced damage of DNA and its components: Experiments and theoretical models. Phys. Rep. $2011,508,1-44$.

(2) Ito, T.; Baker, S.; Stickley, C.; Peak, J.; Peak, M. Dependence of the Yield of Strand Breaks Induced by Gamma-DNA on the Physical conditions of Exposure - WaterContent and Temperature. Int. J. of Radiat. Bio. 1993, 63, 289-296.

(3) von Sonntag, C. The Chemical Basis of Radiation Biology; Taylor \& Francis: London, U.K., 1987.

(4) Steenken, S. Purine bases, nucleosides, and nucleotides: aqueous solution redox chemistry and transformation reactions of their radical cations and e- and $\mathrm{OH}$ adducts. Chem. Rev. 1989, 89, 503-520.

(5) Cooper, G. M. The Cell: A Molecular Approach; Sinauer Associates: Sunderland (MA), 2000.

(6) Cobut, V.; Frongillo, Y.; Patau, J. P.; Goulet, T. Monte Carlo simulation of fast electron and proton tracks in liquid water - I. physical and physicochemical aspects. Radiat. Phys. Chem. 1998, 3, 229-243.

(7) Pimblott, S. M.; LaVerne, J. A. Production of low-energy electrons by ionizing radiation. Radiat. Phys. Chem. 2007, 76, 1244-1247.

(8) Boudaïffa, B. Resonant Formation of DNA Strand Breaks by Low-Energy (3 to 20eV) Electrons. Science 2000, 287, 1658-1660.

(9) Martin, F.; Burrow, P.; Cai, Z.; Cloutier, P.; Hunting, D.; Sanche, L. DNA Strand Breaks Induced by 0 - 4eV Electrons: The Role of Shape Resonances. Phys. Rev. Lett. 2004, 93, 068101. 
(10) Nguyen, J.; Ma, Y.; Luo, T. Direct observation of ultrafast-electron-transfer reactions unravels high effectiveness of reductive DNA damage. P.N.A.S. 2011, 108, 11778.

(11) Boulanouar, O.; Fromm, M.; Mavon, C.; Cloutier, P.; Sanche, L. Dissociative electron attachment to DNA-diamine thin films: Impact of the DNA close environment on the OH- and O- decay channels. J. Chem. Phys 2013, 139.

(12) Alizadeh, E.; Sanz, A. G. Radiation Damage to DNA: The Indirect Effect of Low-Energy Electrons. J. Phys. Chem. Lett. 2013, 4, 820.

(13) Cho, W.; Michaud, M.; Sanche, L. Vibrational and electronic excitations of H2O on thymine films induced by low-energy electrons. J. Chem. Phys 2004, 121, 11289-11295.

(14) Berdys, J.; Anusiewicz, I.; Skurski, P.; Simons, J. Damage to Model DNA Fragments from Very Low-Energy ( $11 \mathrm{eV}$ ) Electrons. J. Am. Chem. Soc. 2004, 126, 6441-6447.

(15) Simons, J. How do low-energy (0.1-2 eV) electrons cause DNA strand breaks. Acc. Chem. Res. 2006, 39, 772-779.

(16) Gu, J.; Xie, Y.; Schaefer, H. Glycosidic bond cleavage of pyrimidine nucleosides by lowenergy electrons: A theoretical rationale. J. Am. Chem. Soc. 2005, 127, 1053-1057.

(17) Gu, J.; Wang, J.; Leszczynski, J. Electron attachment-induced DNA single-strand breaks at the pyrimidine sites. Nucleic Acids Res. 2010, 38, 5280-90.

(18) Liang, G.; Bao, X.; Gu, J. The Possibility of the Decomposition of 2'-Deoxyribose Moiety of Thymidine Induced by the Low Energy Electron Attachment. J. Comput. Chem. 2008, 29, 2648-2655.

(19) Jordan, K. D.; Voora, V. K.; Simons, J. Negative electron affinities from conventional electronic structure methods. Theor. Chem. Acc. 2014, 133, 1-5.

(20) Walch, S. P. Model calculations of the electron affinities and ionization potentials of DNA. Chem. Phys. Lett. 2003, 374, 496-500. 
(21) Tennyson, J. Electron-molecule collision calculations using the R-matrix method. Phys. Rep. 2010, 491, $29-76$.

(22) Tennyson, J. Private communication. 2015.

(23) Williamson, A. The effects of dissociative electrons in different environments. M.Sc. thesis, School of Mathematics and Physics, Queen's University Belfast, 2014.

(24) Gu, J.; Xie, Y.; Schaefer, H. Guanine nucleotides: base-centered and phosphatecentered valence-bound radical anions in aqueous solution. J. Phys. Chem. B 2010, $114,1221-4$.

(25) Gu, J.; Xie, Y.; Schaefer, H. Electron attachment to nucleotides in aqueous solution. Chem. Phys. Chem. 2006, 7, 1885-7.

(26) Smyth, M.; Kohanoff, J.; Fabrikant, I. I. Electron-induced hydrogen loss in uracil in a water cluster environment. J. Chem. Phys 2014, 140.

(27) Schyman, P.; Laaksonen, A. On the effect of low-energy electron induced DNA strand break in aqueous solution: A theoretical study indicating guanine as a weak link in DNA. J. Am. Chem. Soc. 2008, 130, 12254-12255.

(28) Cauët, E.; Bogatko, S.; Liévin, J.; De Proft, F.; Geerlings, P. Electron-attachmentinduced DNA damage: Instantaneous strand breaks. J. Phys. Chem. B 2013, 117, 9669-9676.

(29) Smyth, M.; Kohanoff, J. Excess Electron Interactions with Solvated DNA Nucleotides: Strand Breaks Possible at Room Temperature. J. Am. Chem. Soc. 2012, 134, 91229125.

(30) Smyth, M.; Kohanoff, J. Excess electron localization in solvated DNA bases. Phys. Rev. Lett. 2011, 106, 1-4. 
(31) Gu, J.; Wang, J.; Rak, J.; Leszczynski, J. Findings on the electron-attachment-induced abasic site in a DNA double helix. Angew. Chem. Int. Ed. 2007, 46, 3479-3481.

(32) VandeVondele, J.; Krack, M.; Mohamed, F.; Parrinello, M.; Chassaing, T.; Hutter, J. Quickstep: Fast and accurate density functional calculations using a mixed Gaussian and plane waves approach. Comput. Phys. Commun. 2005, 167, 103-128.

(33) Perdew, J. P.; Burke, K.; Ernzerhof, M. Generalized Gradient Approximation Made Simple. Phys. Rev. Lett. 1996, 77, 3865-3868.

(34) Goedecker, S.; Teter, M.; Hutter, J. Phys. Rev. B

(35) Sprik, M.; Ciccotti, G. Free energy from constrained molecular dynamics. J. Chem. Phys 1998, 109, 7737.

(36) Leszczynski, Jerzy and Close, D. M. Computational Chemistry: Reviews of Current Trends, 8th ed.; World Scientific: London, U.K., 2003.

(37) Wang, C.-R.; Nguyen, J.; Lu, Q.-B. Bond Breaks of Nucleotides by Dissociative Electron Transfer of Nonequilibrium Prehydrated Electrons: A New Molecular Mechanism for Reductive DNA Damage. J. Am. Chem. Soc. 2009, 131, 11320-11322.

(38) Naumov, S.; von Sonntag, C. The Energetics of Rearrangement and Water Elimination Reactions in the Radiolysis of the DNA Bases in Aqueous Solution (eaq and OH Attack): DFT Calculations. Radiat. Res. 2008, 169, 355-363.

(39) Steenken, S.; Telo, J. P.; Novais, H. M.; Candeias, L. P. One-Electron-Reduction Potentials of Pyrimidine-Bases, Nucleosides, and Nucleotides in Aqueous-Solution - Consequences for DNA Redox Chemistry. J. Am. Chem. Soc. 1992, 114, 4701-4709.

(40) Swarts, S. G.; Gilbert, D. C.; Sharma, K. K.; Razskazovskiy, Y.; Purkayastha, S.; Naumenko, K. A.; Bernhard, W. A. Mechanisms of Direct Radiation Damage in DNA, 
Based on a Study of the Yields of Base Damage, Deoxyribose Damage, and Trapped Radicals in d(GCACGCGTGC)(2). Radiat. Res. 2007, 168, 367-381.

(41) Sanche, L. Biological chemistry: Beyond radical thinking. Nature 2009, 461, 358-359.

(42) Wang, C.; Lu, Q. Molecular Mechanism of the DNA Sequence Selectivity of 5-Halo-2 -Deoxyuridines as Potential Radiosensitizers. J. Am. Chem. Soc. 2010, 132, 1471014713.

(43) Zheng, Y.; Wagner, J.; Sanche, L. DNA Damage Induced by Low-Energy Electrons: Electron Transfer and Diffraction. Phys. Rev. Lett. 2006, 96, 208101.

(44) Gu, J.; Wang, J.; Leszczynski, J. Comprehensive analysis of DNA strand breaks at the guanosine site induced by low-energy electron attachment. Chem. Phys. Chem. 2010, $11,175-81$.

(45) Barrios, R.; Skurski, P.; Simons, J. Mechanism for Damage to DNA by Low-Energy Electrons . J. Phys. Chem. B 2002, 106, 7991-7994.

(46) Zheng, Y.; Cloutier, P.; Hunting, D. J.; Sanche, L.; Wagner, J. R. Chemical basis of DNA sugar-phosphate cleavage by low-energy electrons. J. Am. Chem. Soc. 2005, 127, $16592-16598$.

(47) Li, Z.; Cloutier, P.; Sanche, L.; Wagner, J. R. Low-energy electron-induced DNA damage: Effect of base sequence in oligonucleotide trimers. J. Am. Chem. Soc. 2010, 132, $5422-5427$.

(48) Kumar, A.; Sevilla, M. Low-Energy Electron Attachment to 5 -Thymidine Monophosphate : Modeling Single Strand Breaks Through Dissociative Electron Attachment. J. Phys. Chem. B 2007, 111, 5464-5474.

(49) Kumar, A.; Sevilla, M. Role of excited states in low-energy electron (LEE) induced 
strand breaks in DNA model systems: influence of aqueous environment. Chem. Phys.

Chem. 2009, 10, 1426-30.
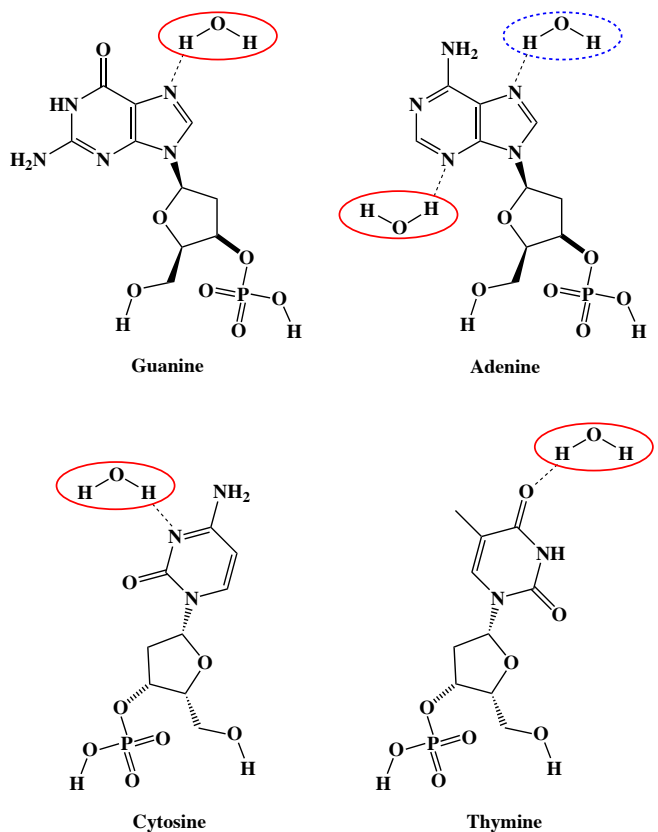

Figure 1: Each of the four nucleotides $\mathrm{dGMPH}^{-}, \mathrm{dAMPH}^{-}, \mathrm{dCMPH}^{-}$and $\mathrm{dTMPH}^{-}$. The nearest water molecules to each base (circled in red) is hydrogen bonded to the site where we protonated each of the bases. When studying the doubly protonated dAMPH ${ }^{--}$we added the second proton to the $\mathrm{N}$-site on the nucleobase that is shown hydrogen bonded to the water molecule circled by a blue dashed line. 


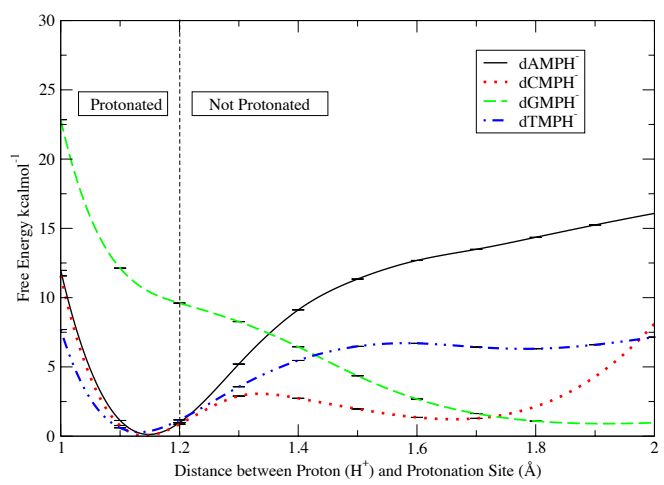

Figure 2: Free energy profiles as a function of the distance between the protonation sites shown in figure 1 and the hydrogen atom belonging to the hydrogen-bonded water molecule calculated from our Blue Moon ensemble simulations. The free energy profiles for $\mathrm{dAMPH}^{-}$, $\mathrm{dCMPH}^{-}, \mathrm{dGMPH}^{-}$and $\mathrm{dTMPH}^{-}$are the solid black line, the dotted red line, the dashed green line and dot-dashed blue line respectively. The average value for the Lagrange multiplier at each constraint value was calculated by averaging over a $1.5 \mathrm{ps}$ simulation. To test convergence we divided these trajectories into four equally long pieces and calculated block averages. The points on the graph show the free energy that was obtained by integrating between these constraints as well as the associated, negligible error bars that were obtained by calculating the standard deviation from our block averages. The vertical dashed line indicates the typical length of a bond involving a hydrogen atom and thus indicates the point at which the nucleobase becomes protonated. It is clear that with the exception of $\mathrm{dGMPH}^{-}$, the free energy of the nucleotides is minimised when it is protonated. 

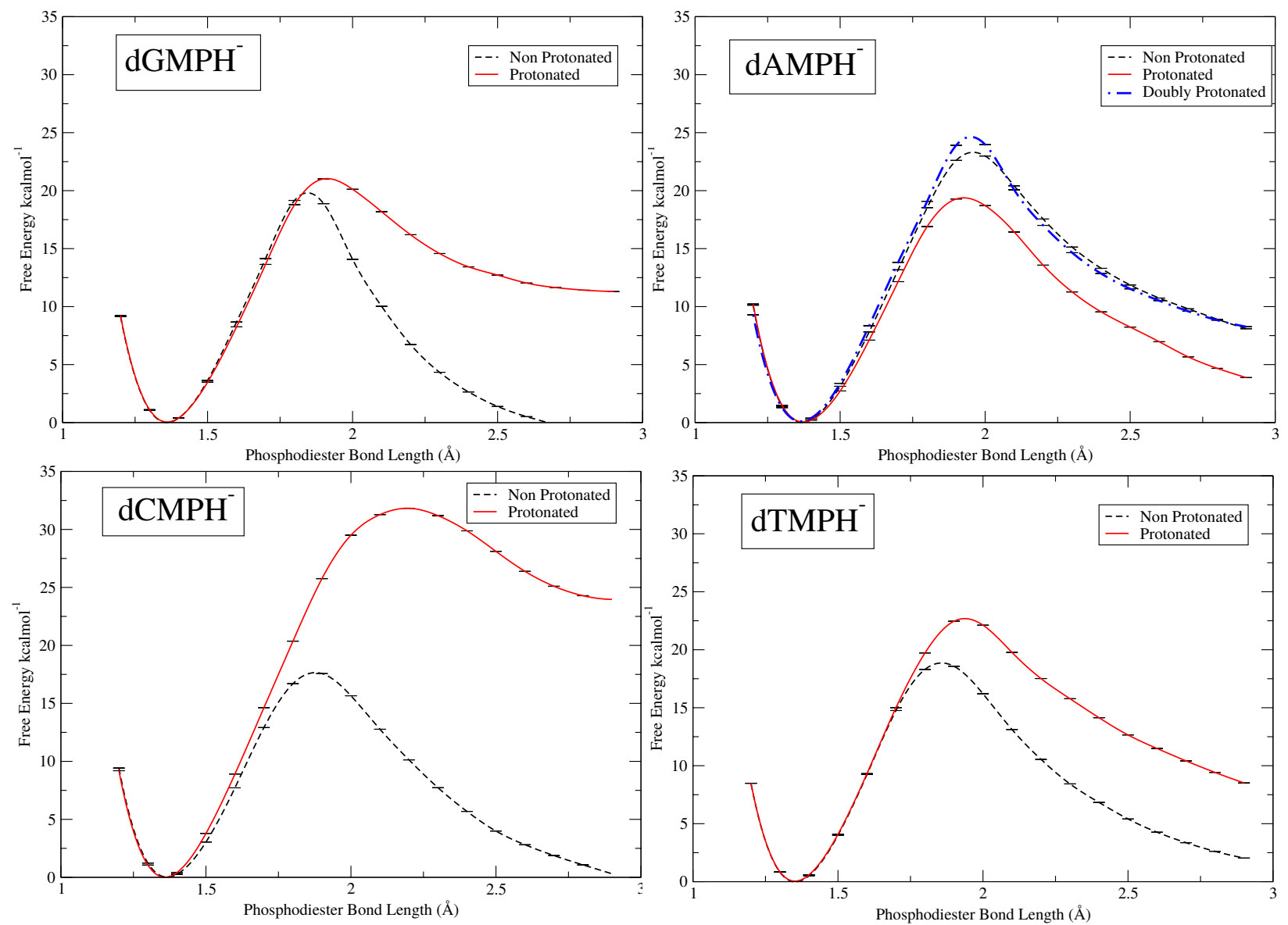

Figure 3: Free Energy profiles as a function of the length of the $\mathrm{C}_{3},-\mathrm{O}_{3}$, (Phosphodiester) bond. The barriers in each of these profiles give a measure of the ease with which this bond is cleaved in the four nucleotides. The free energy profiles in these figures were calculated in the manner described in the caption to figure 2 so error bars are included. Two calculations were performed for each nucleotide one in which an additional restraint was used to ensure that a proton was attached to the site indicated in figure 1 (solid red line) and one in which the site was forced to be deprotonated (dashed black line). It is clear that with the exception of Adenine, the barrier is higher to break the $\mathrm{C}_{3},-\mathrm{O}_{3}$, bond when the base is protonated. Adenine is a special case as it spontaneously protonated during the equilibration of the system, which is in-line with what would be expected given the shape of the protonation free energy profile for this base (see figure 2). For $\mathrm{dAMPH}^{-}$we also studied the doubly protonated base and found that the second protonation increased the height of the barrier (dot dashed blue line in upper right panel). 

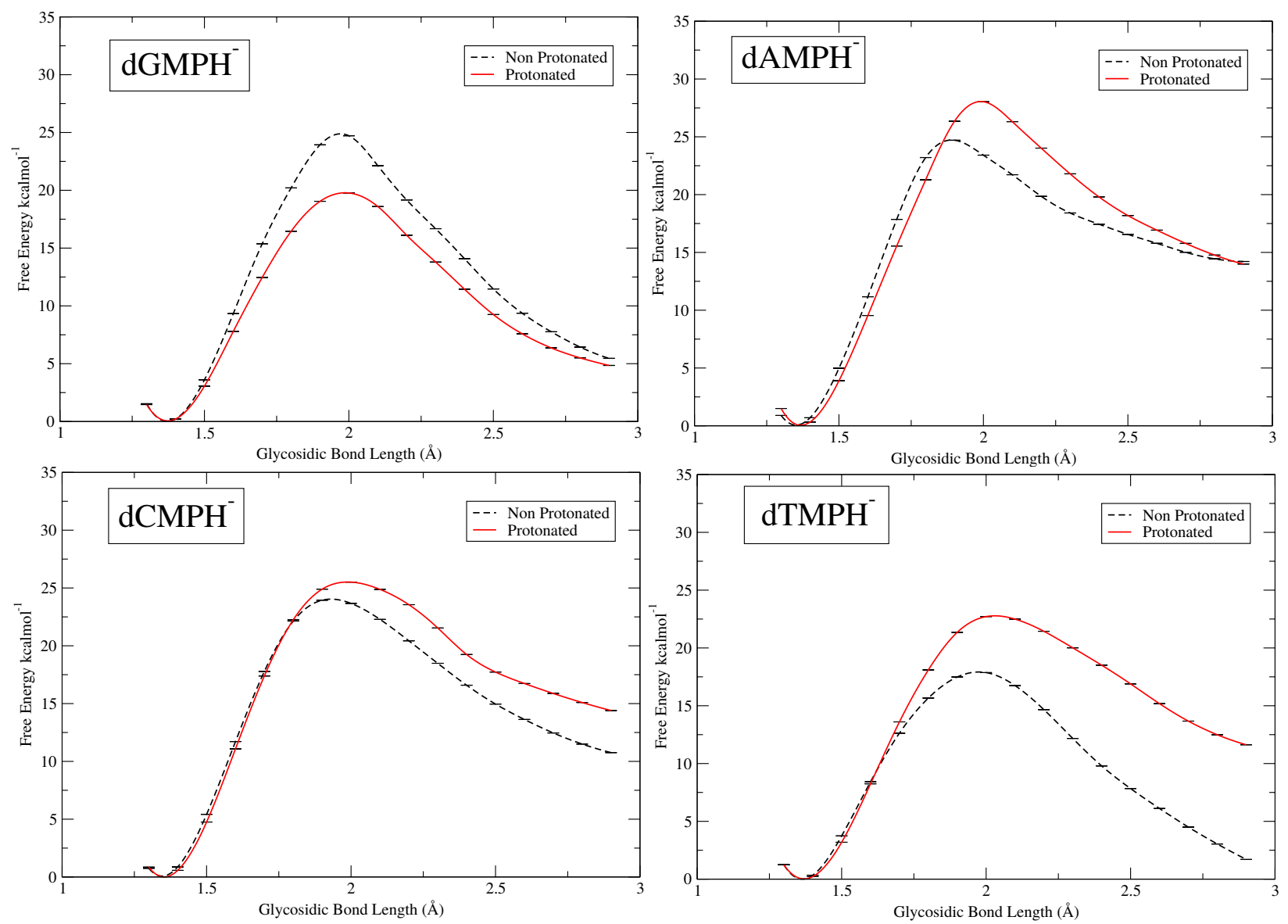

Figure 4: Free Energy profiles as a function of the length of the N-C (Glycosidic) bond. The barriers in each of these profiles give a measure of the ease with which this bond is cleaved in the four nucleotides. The free energy profiles in these figures were calculated in the manner described in the caption to figure 2 so error bars are included. Two calculations were performed for each nucleotide one in which an additional restraint was used to ensure that a proton was attached to the site indicated in figure 1 (solid red line) and one in which the site was forced to be deprotonated (dashed red line). Protonation increases the barrier for glycosidic strand breaking in all bases other than $\mathrm{dGMPH}^{-}$. Comparison of the barriers in this figure with those shown in figure 3 shows that breaking of the glycosidic bond is competitive with breaking the $\mathrm{C}_{3},-\mathrm{O}_{3}$, bond for the pyrimidines $\left(\mathrm{dCMPH}^{-}\right.$and $\left.\mathrm{dTMPH}^{-}\right)$. However, for the purines the barrier to breaking the $\mathrm{C}_{3},-\mathrm{O}_{3}$, bond is energetically less costly than breaking the glycosidic bond. 
Graphical TOC Entry

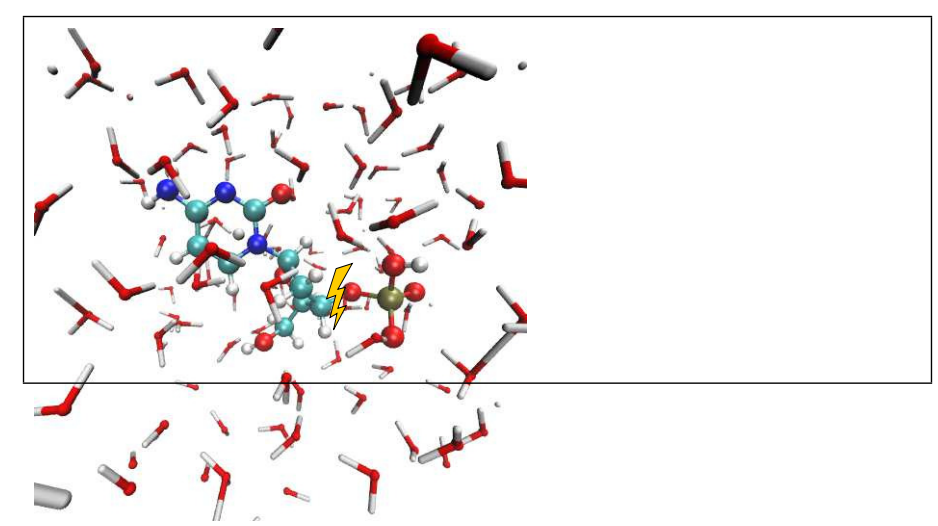

